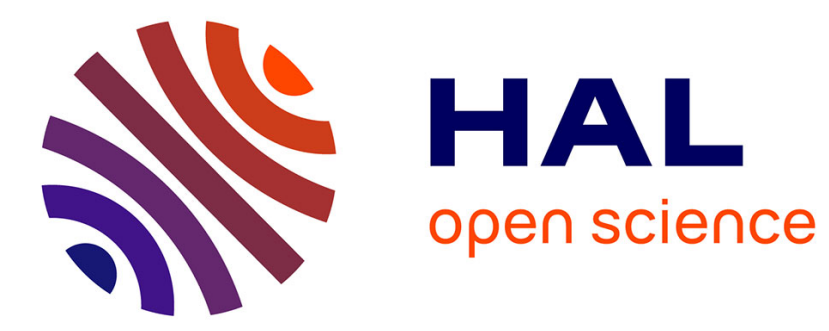

\title{
NEW VISIBLE AND NEAR-INFRARED EMISSION FROM CHEMICALLY GENERATED SINGLET OXYGEN
}

\author{
S. Yoshida, H. Tahil, Y. Hida
}

\section{To cite this version:}

S. Yoshida, H. Tahil, Y. Hida. NEW VISIBLE AND NEAR-INFRARED EMISSION FROM CHEMICALLY GENERATED SINGLET OXYGEN. Journal de Physique IV Proceedings, 1991, 01 (C7), pp.C7-615-C7-618. 10.1051/jp4:19917166 . jpa-00250843

HAL Id: jpa-00250843

https://hal.science/jpa-00250843

Submitted on 1 Jan 1991

HAL is a multi-disciplinary open access archive for the deposit and dissemination of scientific research documents, whether they are published or not. The documents may come from teaching and research institutions in France or abroad, or from public or private research centers.
L'archive ouverte pluridisciplinaire $\mathbf{H A L}$, est destinée au dépôt et à la diffusion de documents scientifiques de niveau recherche, publiés ou non, émanant des établissements d'enseignement et de recherche français ou étrangers, des laboratoires publics ou privés. 


\title{
NEW VISIBLE AND NEAR-INFRARED EMISSION FROM CHEMICALLY GENERATED SINGLET OXYGEN
}

\author{
S. YOSHIDA, H. TAHIL and Y. HIDA \\ Hutech Research Laboratory, 4-27-8 Minami Karasuyama Setagaya, Tokyo 157, Japan
}

\begin{abstract}
New emission in the visible and near infrared based on chemically produced singlet oxygen is discussed. Recent experimental results are presented. Previous research is reviewed and discussed as well.
\end{abstract}

\section{1.- Introduction}

This paper discusses the new visible emission that we happened to discover a couple of years ago in a flow of chemically generated singlet oxygen. The most intriguing aspect of this emission is that it has shown some lasing-like phenomena, implying a new chemical laser in the visible $[1,2]$. But these phenomena are poorly reproducible, unfortunately, and a really coherent laser beam has not been obtained. Moreover, some fundamental matters about the emission including the identification of the emitting species are still up in the air, though it is obviously true that singlet oxygen is the energy source of the emission.

The present visible emission was first observed in the active region of our chemical oxygen iodine laser (COIL) [3] where iodine was injected into the flow of singlet oxygen. At that time the iodine content was the key to increase the emission intensity. But later we found that iodine was not essential for the emission, but certain types of heated metals or metal containing compounds have the same effect showing the identical spectrum [4]. This implies that the present emission is due to a catalytic reaction by those metals / compounds. Thus in this paper, these metals / compounds are referred to as a 'catalyst', though it has not been revealed that the reaction is truly catalytic. In addition, we have found that the visible emission is always accompanied by near infrared emission having major peaks at 1090, 1275, and $1550 \mathrm{~nm}$ in the spectrum [5]. These peak energies correspond to electronic-vibrational transitions of singlet oxygen to the ground state [6].

Several investigators have observed the same emission. Bacis et al. [7] carried out spectroscopic investigation using $\mathrm{Cu}, \mathrm{Al}, \mathrm{Au}$ as the catalyst. Zhuang et al. [8] have observed the emission in a system where singlet oxygen was generated alternatively by the chemical method or a microwave discharge. They measured the radiative lifetime of the red emitter to be $5.4 \mathrm{msec}$. Huang et al. [9] have observed the same visible emission using various metalic catalysts claiming that surface-catalyzed reactions are responsible for initiating the creation of the emitter.Tokuda et al. [10] successfully observed the same emission in a microwave generated-singlet oxygen / $\mathrm{CuCl} 2$ system and concluded that $\mathrm{CuCl} 2$ is the 
emitting species. They have assigined the red emission to $2 \Pi \mathrm{u}-2 \Pi g$ transition of $\mathrm{CuCl} 2$. More recently, Bacis et al. [11] performed a similar experiment and compared the spectrum to the LIF spectrum of $\mathrm{CuCl}$. Consequently, they have made an assignment similar to that of Tokuda et al. [10] Lasing action has not been observed by any of these investigators.

\section{2.- Experimental}

The present emission was observed in a remodeled version of our COIL system where the iodine injector was replaced by a catalyst. For metal catalysts in the form of a wire or tube, an electrical bar heater was used to heat the catalyst. For powdery catalyst, an injector similar to the iodine injector in the $\mathrm{COIL}$ was used. The visible and infrared emissions were monitored by $\mathrm{Si}$ and $\mathrm{PbS}$ detectors, respectively. Details about the system are described in Ref.4.

\section{3.- Emission spectra}

As Fig. 2 of Ref. 4 shows, the visible emission spectrum has an envelope of about 2000 wavenumbers full width at half maximum (FWHM) and many progression like peaks. The highest peak is found around $710 \mathrm{~nm}$, which is almost at the center of the envelope. On the red side of this, there are five peaks of $200-400$ wavenumbers FWHM with about 360 wave number interval. On the blue side, more peaks of a similar width are seen with a smaller interval ( $\sim 100$ wavenumbers). The same spectrum has been observed by other investigators [7 - 10].

Assuming that these peaks are due to vibrational transitions, an attempt was made to arrange the peak energies as a function of an arbitrary vibration number. If all the peak energies are arranged in series, the resulting curve is not linear indicating that the peaks do not belong to one common electronic transition. Therefore we divided the peaks into groups in a way that the peaks in the same group show linearity against the arbitrary vibration number when they are arranged in order of energy. After trying many ways of grouping we have found that linearity is seen when the peaks are divided into two groups in the way shown in Fig.1.

The infrared emission spectrum, on the other hand, consists of three major peaks appearing at 1090,1275 , and $1550 \mathrm{~nm}$. Interestingly, these peak energies correspond to the transition between singlet and ground states of oxygen accompanied by vibrational changes[6] indicating that the emission energy is originally from singlet oxygen. Similar to the visible spectrum, the infrared spectrum has many smaller width peaks. Details about the infrared spectrum are described in Ref.5.

As for the peak energy arrangement, the same procedure as the visible emission was carried out for the infrared spectrum. As is the case of the visible spectrum, the peak energies have been found to show linearity when divided into two groups. The resulting lines are also shown in Fig.1.

The slope of Fig. 1 represents the vibrational energy separation. All four curves show a similar slope of about 370 wavenumber, which is very close to the spacing of ground state symmetric stretching mode of $\mathrm{CuCl} 2$. Noting this point, Tokuda et al. [12] carried out spectroscopic investigation on a singlet delta / $\mathrm{CuCl} 2$ system and assigned the observed spectrum to transitions of $\mathrm{CuCl} 2$. The fact that the visible and infrared data show the same slope, together with the fact that both are divided into two groups, indicates that the visible and infrared emission are due to the same emitting species or one of them is an intermediate of the other. 
In Fig.2, the energy separation between two adjacent peaks in curve 2 of Fig.1 is plotted as a function of the peak energy. The energy separation, called $\delta \mathrm{E}$, corresponds to the vibrational energy separation of this electronic state. As the peak energy increases, $\delta \mathrm{E}$ decreases indicating that this progression represents vibrational numbers of the upper electronic state. This is inconsistent with the hypothesis that the $2 \Pi \mathrm{u}$ state of $\mathrm{CuCl} 2$ is excited from the ground state by two singlet molecules [11,12] because even the energy of the lowest vibrational level of the 2 Hu state is higher than twice of the energy of singlet oxygen and thereby it is impossible to excite to higher vibrational levels. It may be the case that a higher state is generated by three singlet oxygen molecules and then this state decays to the $2 \Pi u$ state by a rapid process. This hypothesis agrees with the fact that the slope of visible emission vs singlet delta emission is three suggesting that at least three singlet oxygen molecules seem to be involved. Further study is required to conclude this matter. For the other curves appearing in Fig.1, $\delta \mathrm{E}$ vs peak energy does not distinctly show either a positive or negative slope.

\section{4.- Properties of the emission}

We randomly chose various materials and examined if they worked as a catalyst for the present emission. Table 1 lists all the materials we examined. Among these materials, $\mathrm{Cu}, \mathrm{Al}, \mathrm{CuCl}$, and $\mathrm{CuCl} 2$ showed stable emission which could last as long as the singlet oxygen was fed. They showed the emission spectrum. Stainless steel, Ni, chromel-alumel (thermocouple) also showed intensified emission, but since the emission generated by these metals was lived too short, typically a few seconds, we could not measure the emission spectrum.

In any case when the intensified emission was observed, the temperature was the key. $\mathrm{Cu}$ and $\mathrm{Cu}$ containing compounds started to intensify the emission remarkably when the temperature reached around 200 centigrade and beyond that point the intensity increased almost proportionally to the temperature. Below 200 centigrade, the present spectrum was not observed but, instead, the spectra of singlet sigma and the so-called dimol emission of singlet oxygen were observed with much lower intensity. A similar tendency was observed by Bacis et al.[7]. Al showed an interesting behavior[5]. When the temperature reached around 200 centigrade, it started the intensification, but the intensity went down quickly unless we kept increasing the temperature. We tested the temperature dependence experiment as high as 400 centigrade. Within this range $\mathrm{Cu}$ showed the highest intensity. $\mathrm{Al}$ and the $\mathrm{Cu}$ containing compounds showed the maximum intensity, an order of magnitude lower than $\mathrm{Cu}$. Polished $\mathrm{Cu}$ showed higher intensity than oxidized $\mathrm{Cu}$.

Simlilar experiments have been carried out by other investigators [7 - 12]. It has been revealed that a wide variety of materials are able to work as a catalyst. These materials include $\mathrm{W}, \mathrm{Sn}$-coated $\mathrm{Cu}$, Nichrome, Pt, and Ag, in addition to those listed in Table 1. It should be emphasized that all the materials have shown the same emission spectrum. Whatever is the identity of the emitting species, there is a consensus among all of the investigators: i.e.,(i) emission energy is probably originally from singlet oxygen, (ii) $\mathrm{Cl} 2$ gas is needed, and (iii) some catalyst is prerequisite.

While the fact that the emission spectrum does not depend on the choice of the catalyst implies catalytic reaction takes place on the surface, there are observations which imply gas phase reactions. Often we observed a dark zone between the catalyst and the red emitting area. The same phenomenon has been observed by Zhuang [8] and Bacis [7]. More mysteriously, we sometimes observed the red emission even upstream from the catalyst. Huang [9] has observed the emission upstream too. The reason for the upstream emission is not clear at present. 


\section{5.- Conclusion}

Since the first observation of the present emission, many matters have been revealed. However, many questions still remain unsolved. As far as the assignment of the emission spectrura to known transitions is concerned, $\mathrm{CuCl} 2$ seems most probably to be the emitting species. But if does not explain the big question: Why is the spectrum independent of the choice of the catalyst? In addition litte has been understood about the energy transfer mechanism. Further intensive spectroscopic research is of great necessity to pursue investigation on lasing.

\section{Acknowledgement}

A part of the present investigation was carried out in the IRI Laser Laboratory.

\section{References}

1. S.Yoshida, et al. Jap. J. Appl. Phys., 28, L831 (1989)

2. S.Yoshida, et al.Apple. Phys. Lett. 54, 2400 (1989)

3. K. Shimizu, et al. J. Appl. Phys. 69, 79 (1991)

4. S.Yoshida, et al. Appl. Phys. Lett. 55, 2707 (1989)

5. S. Yoshida, et al. Appl. Phys. Lett. 57, 645 (1990)

6. D. L. Huestis, et al. J. Chem. Phys. 60, 4471 (1974)

7. R.Bacis, et al. Europhys. Lett. 12, 569 (1990)

8. Qi.Zhuang, et al. presented at the Conference on Laser and Electro-Optics, May 20-25, 1990, Annaheim, Ca. USA

9. R.Huang, et al. Chem. Phys. Lett. 170, 437 (1990)

10. T.Tokuda, private communication (1991)

11. R.Bacis, Private communication (1991)

12. T.Tokuda, et al. Chem. Phys. Lett. 174, 385 (1990)

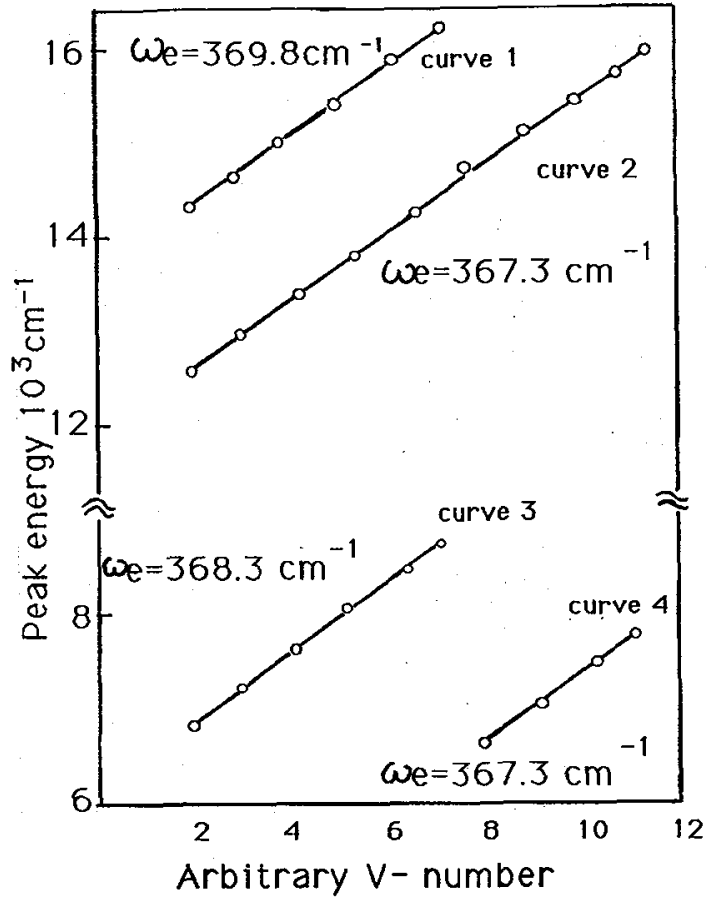

Fig1. Peak energy vs vibration number
Table 1: Materials tested as a catalyst

\begin{tabular}{|l|l|}
\hline \multicolumn{1}{|c|}{ Material } & \multicolumn{1}{c|}{ Emission } \\
\hline Cu (tube / powder) & stable, intense \\
$\mathrm{Al}$ (tube) & stable, medium \\
Stainless steel (bar) & unstable, weak \\
Carbon Steel (bar) & not observed \\
Chromel (wire) & unstable, weak \\
Alumel (wire) & unstable, weak \\
$\mathrm{Ni}$ (wire) & unstable, weak \\
$\mathrm{Solder}$ (wire) & not observed \\
$\mathrm{Pt}$ (wire) & not observed \\
$\mathrm{CuCl}$ (powder) & stable, medium \\
$\mathrm{CuCl}$ (powder) & stable, medium \\
$\mathrm{CuO}$ (powder) & not observed \\
$\mathrm{AlCl}$ (powder) & not observed \\
\hline
\end{tabular}

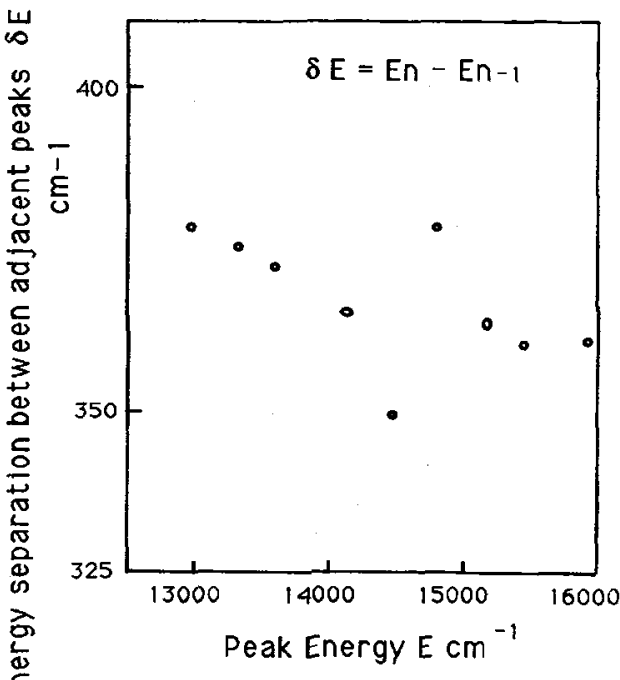

Fig 2. $\delta$ E vs peak energy 